\title{
BIOCHEMICAL AND MORPHOLOGICAL MARKERS OF EXPERIMENTAL SCOPOLAMINE-INDUCED NEURODEGENERATION AND THE EFFECT OF ENALAPRIL ON THEM
}

10.36740/WLek202010102

\author{
Olga G. Kmet, Nataliia D. Filipets, Taras I. Kmet, Yurii M. Vepriuk, Kateryna V. Vlasova \\ BUKOVINIAN STATE MEDICAL UNIVERSITY, CHERNIVTSI, UKRAINE
}

\begin{abstract}
The aim: Was to study enalapril effect on protein peroxide oxidation and lipids of the cerebral cortex and hippocampus of rats with neurodegeneration pattern.

Materials and methods: The experiments were conducted on nonlinear albino male rats $0,18-0,20 \mathrm{~kg}$ of the body weight. Modeled neurodegeneration was simulated by intraperitoneal introduction of scopolamine hydrochloride during 27 days in the dose of $1 \mathrm{mg} / \mathrm{kg}$. Enalapril was introduced intraperitoneal in the dose of $1 \mathrm{mg} / \mathrm{kg}$.

Results: In rats with modeled scopolamine-induced neurodegeneration in the cerebral cortex and hippocampus under enalapril effect, the content of products, reacting with 2-thiobarbituric acid and proteins of a neutral and major character, decreases, which is indicative of a reduced intensity of lipid and protein peroxide oxidation. Morphologic the number of cells with karyopyknosis signs decreases and a relative staining density of the neuron tigroid substance increases, which is indicative of inhibition of the progress of the cerebral neurodestructive processes under conditions of scopolamine-induced damage.

Conclusions: The obtained results are indicative of enalapril ability to improve the examined indices, which is the evidence of increased processes of antioxidant protection and possibility to inhibit neurodegeneration development in case of scopolamine induced lesion of the brain.
\end{abstract}

KEY WORDS: scopolamine-induced neurodegeneration, enalapril, protein and lipid peroxide oxidation

Wiad Lek. 2020;73(10):2114-2119

\section{INTRODUCTION}

Neurodegenerative diseases are characterized by progressive damage of nerve cells and loss of neurons resulting in disorders of the motor and cognitive functions. Pathophysiological mechanisms of neurodegenerative processes including lateral amyotrophic sclerosis, Parkinson's disease, Alzheimer's disease, are not completely found out. Numerous scientific reports today indicate high levels of oxygen reactive forms in the brain of patients with neurodegenerative diseases and indicate a critical role of oxidative stress among the factors inducing neurodegeneration [1].

Neuron cells are highly sensitive to oxidative stress since their functional activity considerably depends on oxidative phosphorylation - a process of energy production for metabolic processes. Therefore, the study of protein peroxide oxidation (PPO) and lipid peroxide oxidation (LPO) in the cerebral cortex and hippocampus of rats is essential to develop a strategy of prevention and treatment of neurodegenerative processes associated with oxidative stress [2].

For many years renin-angiotensin system (RAS) blockers have been effectively used in the treatment of cardio-vascular pathology. Recently such pleiotropic effect of RAS as intensification of cognitive functions has been found [3]. RAS activation is known to intensify oxidative damage of the cellular membranes due to increase of reactive oxygen forms. It results in oxidation of cellular structures and tissue damage [4]. In this respect the efficacy of administration of RAS inhibitors as medicinal agents with pronounced poly-protective properties should be mentioned here. According to literary data central angiotensin converting enzyme (ACE) plays an important role in the process of cerebral microcirculation, metabolism and is a leading factor in the mechanisms of ischemic lesion of neurons and neuron inflammation. RAS inhibition is evidenced to be an effective pathogenic direction to reduce the progress of neuron structural rebuilding after acute cerebral circulatory disorders [5].

\section{THE AIM}

To study enalapril effect on protein peroxide oxidation and lipids of the cerebral cortex and hippocampus of rats with neurodegeneration pattern.

\section{MATERIALS AND METHODS}

The experiments were conducted on nonlinear albino male rats with the body weight of $0,18-0,20 \mathrm{~kg}$, keeping to the requirements of the European Convention for the Protection of Vertebrate Animals used for Experimental and other Scientific Purposes.

Neurodegeneration pattern was simulated by means of intraperitoneal introduction of scopolamine hydrochloride (Sigma, USA) during 27 days in the dose of $1 \mathrm{mg} / \mathrm{kg}$. 
Table I. Enalapril effect on protein and lipid peroxide oxidation in the cytosolic fraction of the cerebral cortex and hippocampus of rats with scopolamine-induced neurodegeneration $(M \pm m, n=7)$

\begin{tabular}{ccccc}
\hline Indices & $\begin{array}{c}\text { Examined } \\
\text { structures }\end{array}$ & Control & $\begin{array}{c}\text { Neurodegeneration } \\
\text { model }\end{array}$ & $\begin{array}{c}\text { Neurodegeneration model + } \\
\text { enalapril }\end{array}$ \\
\hline $\begin{array}{c}\text { OMP } \lambda=370, \\
\text { units/g of tissue }\end{array}$ & Cerebral cortex & $30,486 \pm 1,162$ & $40,700 \pm 1,888^{*}$ & $35,300 \pm 1,221^{*}, \#$ \\
\cline { 2 - 4 } & Hippocampus & $20,357 \pm 1,649$ & $30,643 \pm 1,860^{*}$ & $25,286 \pm 1,196^{*}, \#$ \\
\hline $\begin{array}{c}\text { OMP } \lambda=430, \\
\text { units/g of tissue }\end{array}$ & Cerebral cortex & $30,514 \pm 0,724$ & $36,457 \pm 0,577^{*}$ & $34,257 \pm 0,770^{*}, \#$ \\
\cline { 2 - 4 } & Hippocampus & $20,514 \pm 0,724$ & $26,471 \pm 0,559^{*}$ & $24,271 \pm 0,752^{*}, \#$ \\
\hline $\begin{array}{c}\text { TBA } \\
\text { Cerebral cortex }\end{array}$ & $43,002 \pm 2,367$ & $82,048 \pm 1,662^{*}$ & $75,349 \pm 2,149^{*}, \#$ \\
\cline { 2 - 4 } Hippocampus & $39,961 \pm 3,107$ & $75,198 \pm 5,327^{*}$ & $58,129 \pm 2,224^{*}, \#$
\end{tabular}

Notes: * - reliability compared with the control, \# - reliability compared with neurodegeneration model.

On the $28^{\text {th }}$ day of the experiment enalapril (Zdorovye, Ukraine) was introduced intraperitoneally in the dose of 1 $\mathrm{mg} / \mathrm{kg}$ in $1 \mathrm{ml}$ of physiological solution [6]. The brain was removed cold, then it was washed with cool $0,9 \% \mathrm{NaCl}$ solution, and the cerebral cortex and hippocampus were isolated according to coordinates of the stereotaxic atlas $[7,8]$. Cytoplasmic fraction was isolated by means of the method of differentiation centrifugation of blood homogenates taken from the cerebral cortex and hippocampus on the refrigerator centrifuge at $1000 \mathrm{~g}$ per $10 \mathrm{~min}$., followed by $-1400 \mathrm{~g}$ per $10 \mathrm{~min}$., at the temperature of $4{ }^{\circ} \mathrm{C}$.

Content OMP (oxidation modification protein) in homogenates was determined by the amount of their oxidation modification products by means of spectrophotometry method with the wave length of 370 and $430 \mathrm{~nm}$. The method is based on the reaction of interaction of oxidized amino acid protein residues with 2,4-dinitrophenylhydrazine with the formation of its derivatives, which optic density was determined by means of spectrophotometry. Aldehyde- or ketone- derivatives of a neutral or the main character possessing different ranges of absorption spectrum are known to be formed resulting from protein oxidation depending on amino acids of a neutral (valine, leucine, isoleucine, etc.) or main (lysine, arginine, etc.) character prevailing in their molecules. With $\lambda=370 \mathrm{~nm}$ ketone dinitrophenylhydrazones of a neutral character are determined, with $\lambda=430 \mathrm{~nm}$ - aldehyde dinitrophenylhydrazones of the main character [9] OMP content was expressed in the units per gram of the tissue. Intensity of LPO was evaluated by the content of products reacting with 2-thiobarbituric acid (TBA), which amount was calculated in mcmol per 1 gram of the tissue [10].

The brain samples for histological examination were fixed in $10 \%$ neutral formalin solution, and after the standard histologic processing the tissue was embedded in paraffin. Paraffin histological sections of the cerebral cortex and hippocampus tissues $5 \mathrm{mcm}$ thick were prepared by means of a sliding microtome MC-2. After deparaffinization certain specimens were stained with hematoxylin and eosin, others - with neutral red by Nisl method in order to find tigroid substance [11].
Microslides were examined under the light microscope. Digital copies of optic images were obtained by means of the digital camera Olympus SP550UZ and analyzed by means of the specialized computer program ImageJ for histologic studies $(1.48 \mathrm{v}$, free License, W. Rasband, National Institute of Health, USA) [12].

The obtained results were processed by means of Student $\mathrm{t}$-criterion. Mann-Whitney criterion was used parallel which demonstrated similar results. Therefore, reliability level was considered to be sufficient with $\mathrm{p} \leq 0,05$.

\section{RESULTS}

In comparison with the control group in rats with scopolamine induced lesion of the brain the content of proteins of a neutral character in the cerebral cortex and hippocampus 33,5 and 50,5\% increased, and proteins of the basic character 19,5 and 28,9\% respectively (Table). Increased degree of PPO in the examined homogenates is indicative of protein damage in the brain under conditions of scopolamine introduction. At the same time, in rats with neurodegeneration pattern the amount of TBA in the cerebral cortex and hippocampus increased 47,6 and $46,9 \%$ compared to the control group.

After administration of enalapril to rats with neurodegeneration pattern PPO in the cerebral cortex and hippocampus, registered at $\lambda=370 \mathrm{~nm}$, decreased 13,3 and $17,5 \%$ respectively. POM registration at $\lambda=430 \mathrm{~nm}$ detected on an average $7,2 \%$ increase of the content of neutral and main character proteins in both structures of the brain under enalapril effect.

TBA content in rats with neurodegeneration after enalapril introduction remained higher than that of the control. Though, concerning the untreated group, TBA content decreased in the cerebral cortex $8,2 \%$ and in the hippocampus - $22,7 \%$.

In histological specimens of the control group of rats the neurons with karyopyknosis signs were not found (Fig.1a, $1 b, 4 a, 4 b)$. 


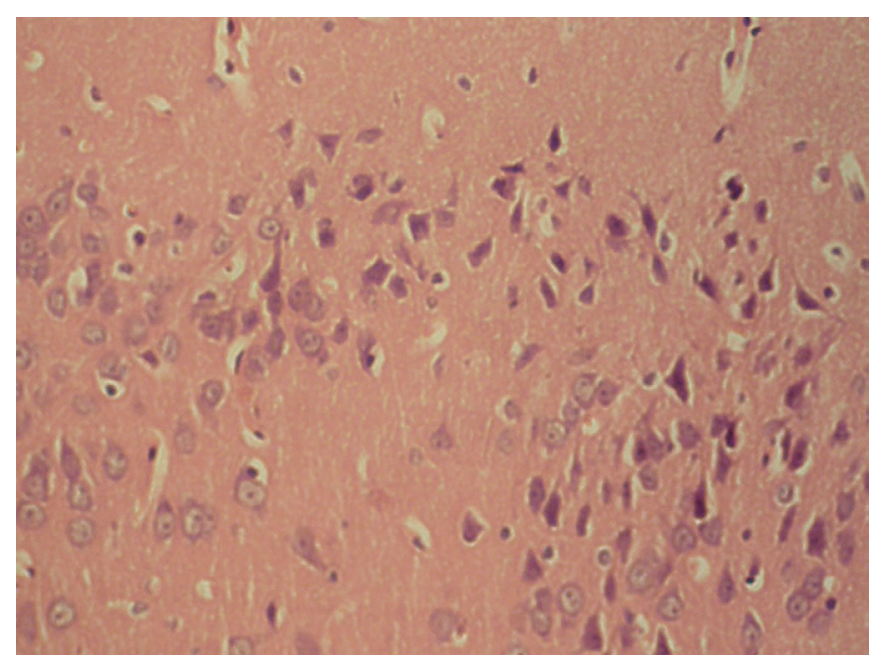

Fig.1a. Cerebral cortex of the control group of rats ( 200$)$ : hematoxylin-eosin.

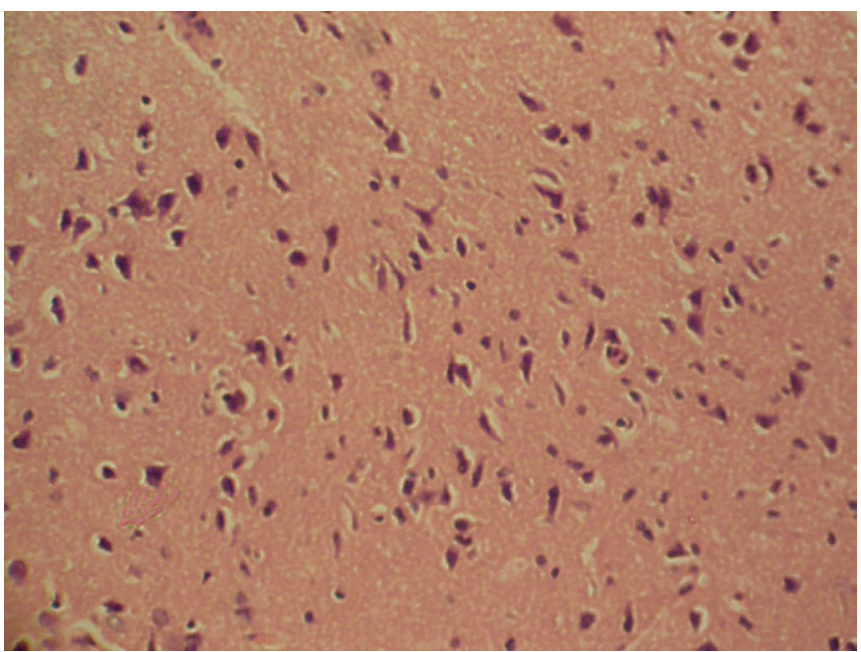

Fig.2a.Cerebral cortex of rats with scopolamine-induced neurodegeneration (²00): hematoxylin-eosin.

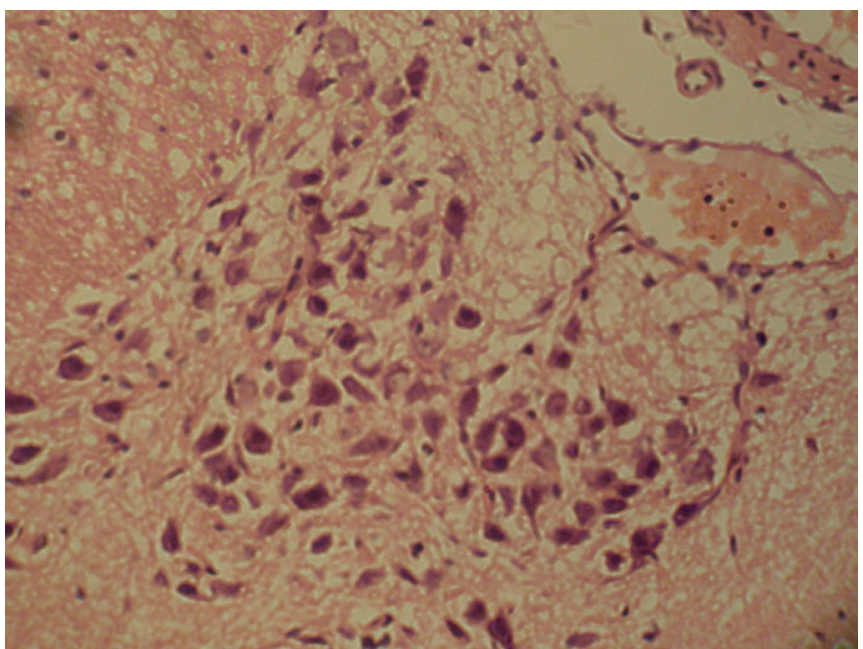

Fig.3a.Cerebral cortex of rats with scopolamine-induced neurodegeneration under conditions of enalapril administration ('200): hematoxylin-eosin.
Fig.1b. Cerebral cortex of the control group of rats ('200): neutral red by Nisl.

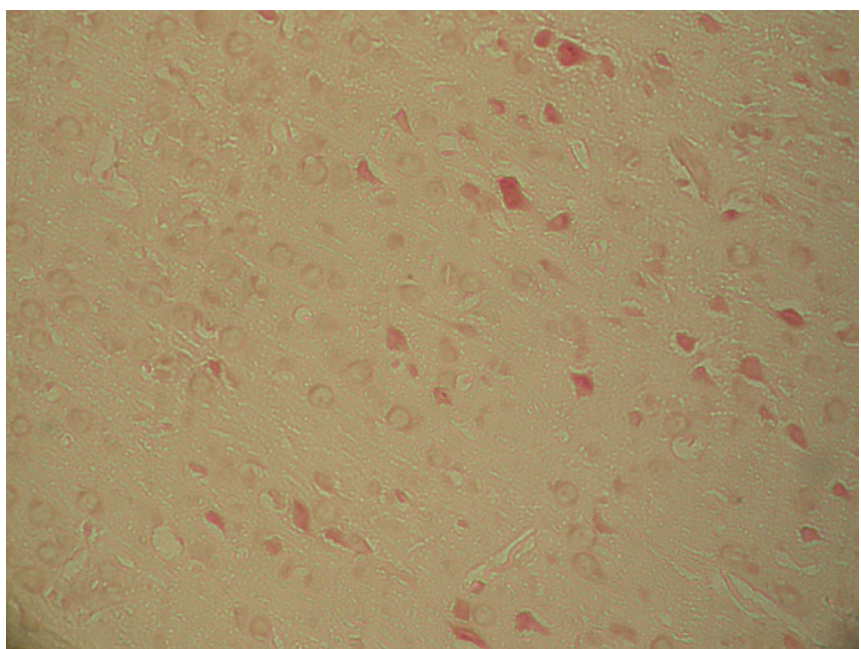

Fig.2b. Cerebral cortex of rats with scopolamine-induced neurodegeneration (²00): neutral red by Nisl.

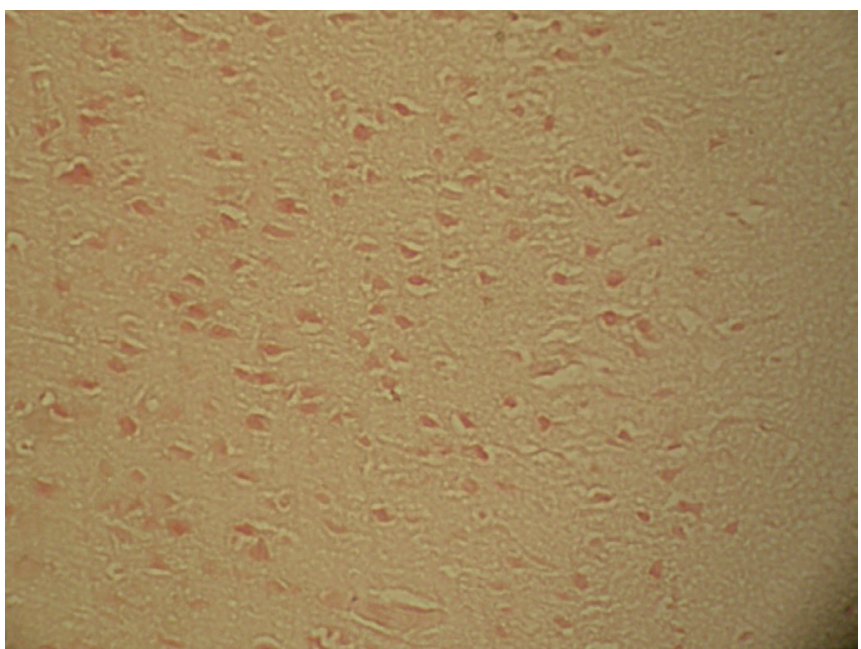

Fig.3b. Cerebral cortex of rats with scopolamine-induced neurodegeneration under conditions of enalapril administration ( $(200)$ :neutral red by Nisl. 


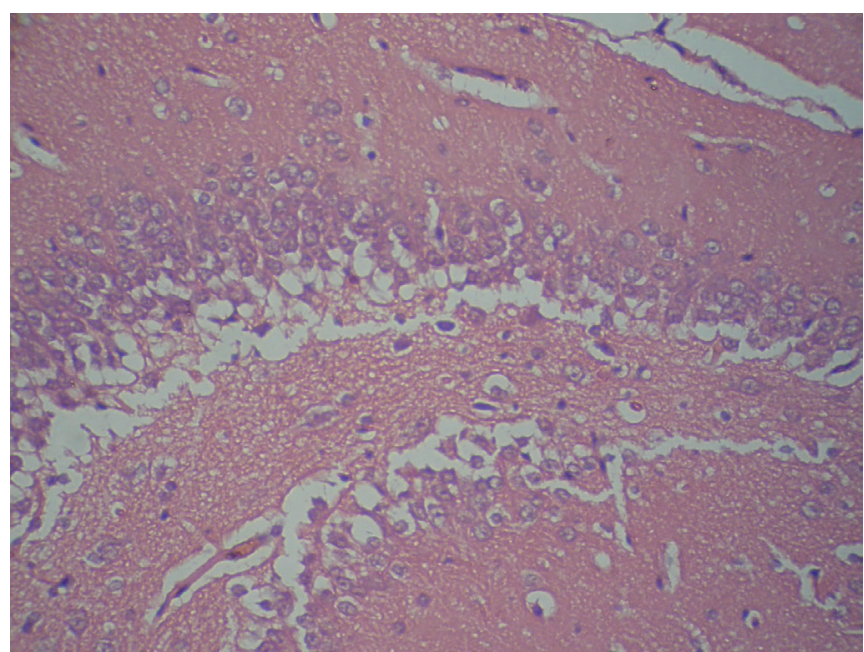

Fig.4a. Hippocampus of the control group of rats ( 200$)$ : hematoxylin-eosin.

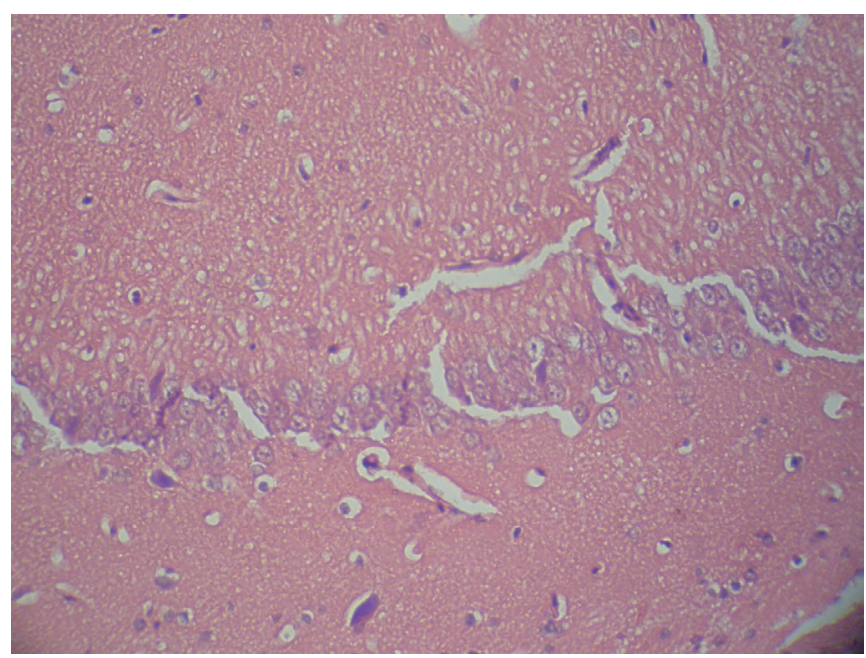

Fig.5a. Hippocampus of rats with scopolamine-induced neurodegeneration (²00): hematoxylin-eosin.

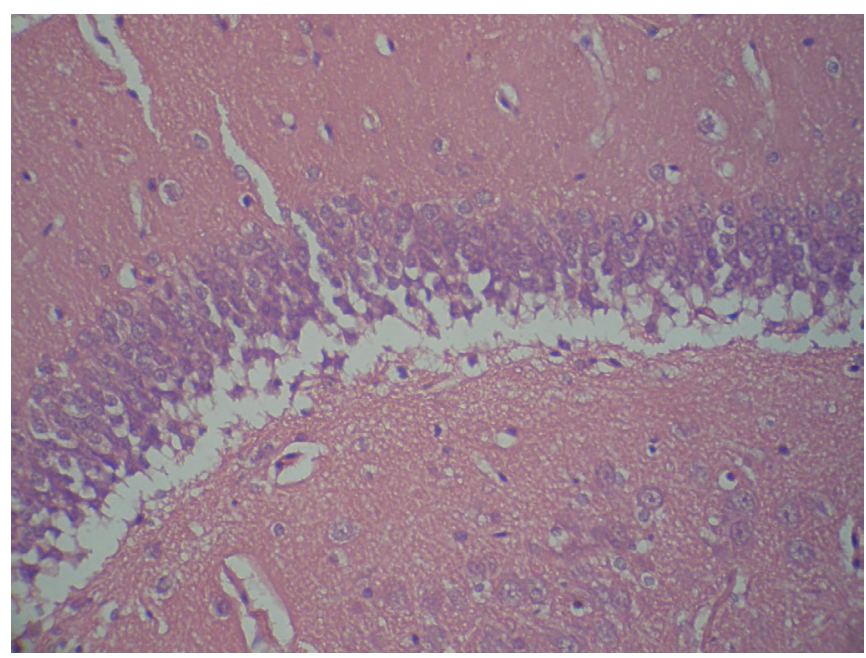

Fig.6a. Hippocampus of rats with scopolamine-induced neurodegeneration under conditions of enalapril administration ( $(200)$ : hematoxylin-eosin

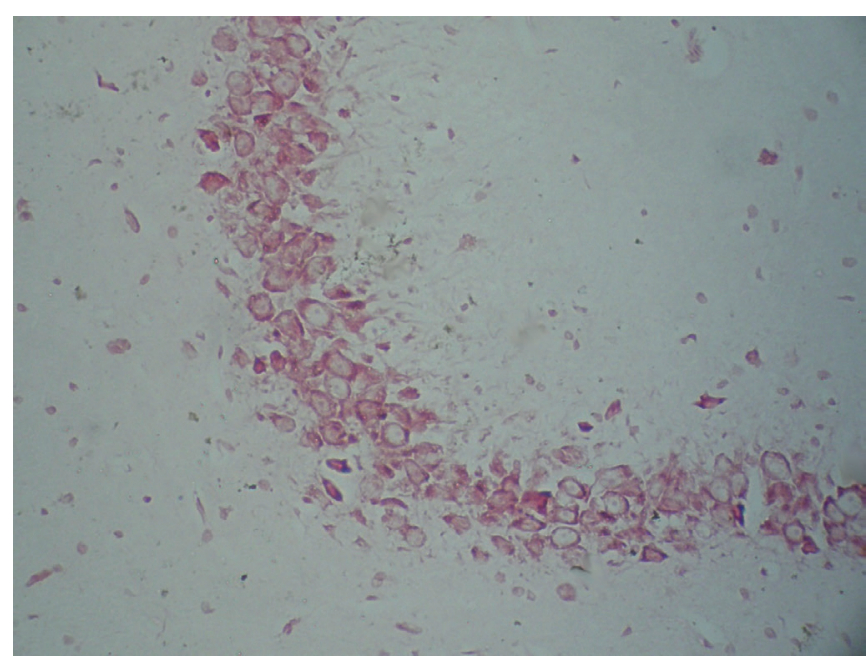

Fig.4b. Hippocampus of the control group of rats ( 200$)$ : neutral red by Nisl.

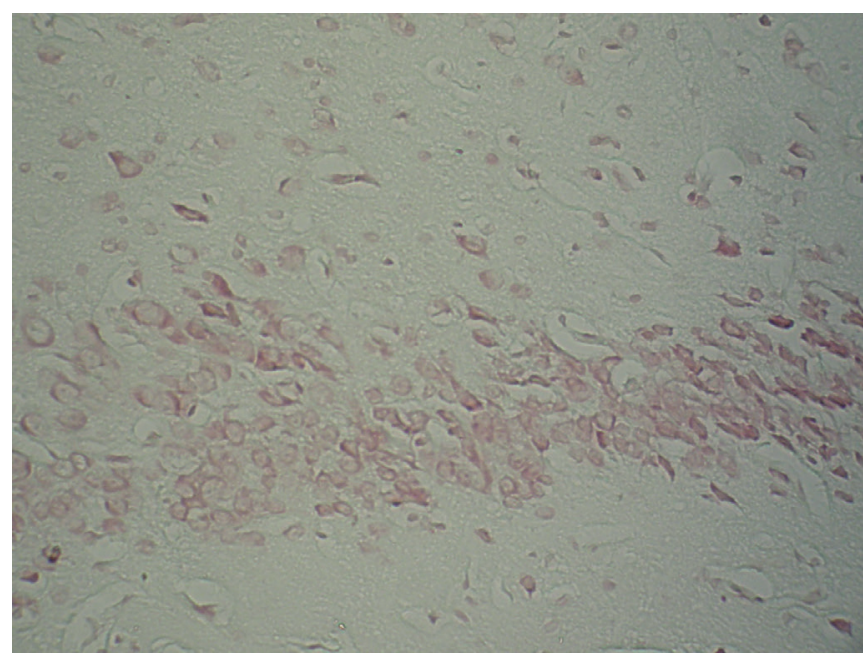

Fig.5b. Hippocampus of rats with scopolamine-induced neurodegeneration (²00): neutral red by Nisl.

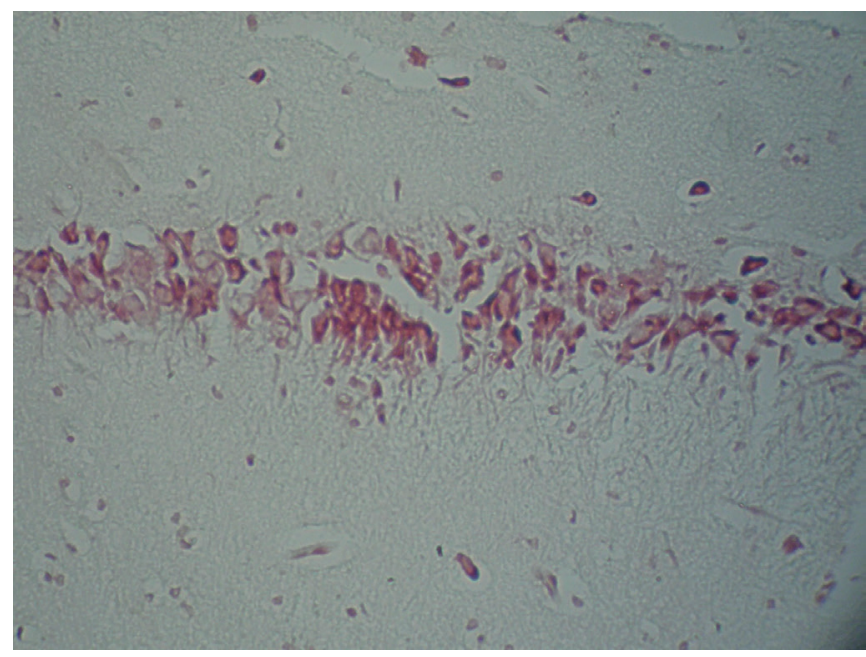

Fig.6b. Hippocampus of rats with scopolamine-induced neurodegeneration under conditions of enalapril administration ( $(200)$ : neutral red by Nisl. 
Against the ground of scopolamine-induced neurodegeneration the neurons with karyopyknosis signs were found compared with the control rats (in 6,9 $\pm 0,18 \%$ - in the cerebral cortex; in 7,2 $\pm 0,19 \%$ - in the hippocampus). In addition, a relative staining density of the neuron tigroid substance in the cerebral cortex and hippocampus was twice decreased as much (Fig. 2a, 2b, 5a, 5b).

Plaque-like formations of homogenous or fibrous structure of various sizes are found in the white substance. Separate fine calcifications are seen near by. After administration of enalapril, the number of cells with karyopicnose decreased 1,8 times in the cerebral cortex and 1,7 times in the hippocampus, and the relative density of the neuronal substance increased by $33.6 \%$ and $37 \%$, respectively (Fig. $3 \mathrm{a}, 3 \mathrm{~b}, 6 \mathrm{a}, 6 \mathrm{~b})$. At the same time the amount of plaque-like formations did not decrease.

\section{DISCUSSION}

One of the causes of increasing concentrations of protein oxidative modification (POM) and TBA in the brain homogenates under conditions of neurodegenerative diseases might be reduced activity of the antioxidant protection system, especially enzymes which are the first to neutralize oxygen active forms, and which is the main reason of activation of protein and lipid peroxide oxidation.

Thus, the results of the study concerning PPO and LPO in the cerebral cortex and hippocampus under conditions of inhibition of the central cholinergic effect produced by scopolamine are indicative of the development of cerebral degeneration. After enalapril was introduced the level of POM and TBA in the cerebral cortex and hippocampus decreased, especially the latter one. The obtained results are indicative of enalapril ability to improve the examined indices, which is the evidence of increased processes of antioxidant protection and possibility to inhibit neurodegeneration development in case of scopolamine induced lesion of the brain.

\section{CONCLUSIONS}

1. In rats with scopolamine induced pattern of neurodegeneration the content of products reacting with 2 -thiobarbituric acid and proteins of the neutral and basic character decrease in the cerebral cortex and hippocampus under enalapril effect, which is indicative of a reduced intensity of lipid and protein peroxide oxidation.

2. Under enalapril effect the number of cells with karyopyknosis signs decreases and a relative staining density of the neuron tigroid substance increases, which is indicative of inhibition of the progress of the cerebral neurodestructive processes under conditions of scopolamine-induced damage.

3. Comprehensive biochemical and morphological investigation is indicative of enalapril correcting effect on protein and lipid peroxide oxidation in the cerebral cortex and hippocampus of rats with scopolamine-in- duced neurodegeneration, which is the evidence of neuroprotective effect under conditions of central cholinergic blockade.

\section{REFERENCES}

1. Kim G.H., Kim J.E., Rhie S.J., Yoon S. The Role of Oxidative Stress in Neurodegenerative Diseases. Exp Neurobiol. 2015;24(4):325-340.

2. Lennicke C., Rahn J., Heimer N. et al. Redox proteomics: Methods for the identification and enrichment of redox-modified proteins and their applications. Proteomics. 2016;16(2):197-213.

3. Jackson L., Eldahshan W., Fagan S.C., Ergul A. Within the Brain: The Renin Angiotensin System. Int J Mol Sci. 2018;19(3):1-23. doi: 10.3390/ ijms19030876.

4. Torika N., Asraf K., Danon A. et al. Telmisartan Modulates Glial Activation: In Vitro and In Vivo Studies. PLOS ONE. 2016;11(5):1-15.

5. Farag E., Sessler D.I., Ebrahim Z. et al. The renin angiotensin system and the brain: New developments. J Clin Neurosci. 2017;46:1-8.

6. Panahpour H., Dehghani G.A., BohlooliS. Enalapril attenuates ischaemic brain oedema and protects the blood-brain barrier in rats via an antioxidant action. Clin Exp Pharmacol Physiol. 2014;41(3):220-226.

7. Paxinos G., Watson C. The Rat Brain in Stereotaxic Coordinates, 7th Edition. Academic Press. 2013;472.

8. Rubin R.D., Watson P.D., Duff M.C., Cohen N.J. The role of the hippocampus in flexible cognition and social behavior. Front Hum Neurosci. 2014;8:1-15.

9. Gerush I.V., Bevzo V.V., Ferenchuk Ye.0. The effect of melatonin on lipid peroxide oxidation, oxidative modification of proteins and mitochondria swelling in the skeletal muscle tissue of rats under alloxan diabetes. Ukr. Biochem. J 2018;90(3):62-69.

10. Kushnir O.Yu., Yaremii I.M., Shvets V.I., Shvets N.V. Influence of melatonin on glutathione system in rats skeletal muscle under alloxan induced diabeteshttps. Fiziol Zh. 2018;64(5):54-62.

11. Venerucci F. Histopathology kits: methods and applications. Bologna, Milan: Bio-Optica. 2016;98

12. Ferreira T., Rasband W. Image J. User Guide. New York. National Institute of Health. 2012;187.

The work was performed in accordance with the planned research of the department of Pathological Physiology of the Higher State Educational Establishment of Ukraine "Bukovinian State Medical University» on the topic: «New methodical approaches to the pathogenetic treatment of dysfunction of the proximal nephron in conditions of the development of dysregulation pathological process of renal and non-renal origin» (state registration number 0118U001193). The Ministry of Health of Ukraine is the institution that finances the research. The authors assumes full responsibility for everything published in the article.

\section{ORCID and contributionship:}

Olga G. Kmet: 0000-0003-0336-1103 A,B,C,D

Nataliia D. Filipets: 0000-0001-8582-6685 ${ }^{\mathrm{A}}$

Taras I. Kmet: 0000-0002-2850-9111 ${ }^{F}$

Yurii M. Vepriuk: 0000-0002-3803-8085 ${ }^{E}$

Kateryna V. Vlasova: 0000-0002-8969-105X

\section{Conflict of interest:}

The Authors declare no conflict of interest. 


\section{CORRESPONDING AUTHOR}

Olga G. Kmet

Bukovinian State Medical University

2 Teatralna Sq., 58002 Chernivtsi, Ukraine

tel: +380958634111

e-mail: kmet.olga@bsmu.edu.ua

Received: 10.10 .2019

Accepted: 31.08 .2020

A - Work concept and design, B - Data collection and analysis, C - Responsibility for statistical analysis,

D-Writing the article, $\mathbf{E}$-Critical review, $\mathbf{F}$ - Final approval of the article 